\title{
A efetividade de dois tratamentos para disfunção temporomandibular muscular: estudo piloto
}

\author{
The effectiveness of two treatments for muscle temporomandibular \\ dysfunction: pilot study
}

\author{
Júlia Pagnussat* \\ Leonardo Saraiva** \\ Aline Avrella*** \\ Larissa Pires Ceron ${ }^{* * * *}$ \\ Fernando Thalheimer Bacchi $i^{* * * * *}$ \\ Carolina Barreto Mozzini*
}

\section{Resumo}

Objetivo: comparar a eficácia de dois protocolos de tratamento para disfunção temporomandibular (DTM) de origem muscular. Sujeitos e método: Estudantes da Faculdade Especializada na Área de Saúde do Rio Grande do Sul foram selecionados por meio de questionário, para avaliar a presença de sinais e sintomas de DTM e diagnóstico de DTM muscular pelos Critérios de Diagnóstico para Transtornos da Pesquisa Temporomandibular. Dez estudantes compuseram a amostra, divididos em dois grupos, de acordo com o protocolo de tratamento: G1 - hipertermia induzida, exercícios mandibulares e massagem; e G2 - agulhamento seco, hipertermia induzida, exercícios mandibulares e massagem. Os alunos foram instruídos a realizar o protocolo do tratamento G1 todos os dias em casa e na instituição de ensino por três sessões, que ocorreram a cada cinquenta dias. $\mathrm{O}$ agulhamento seco foi realizado no G2 apenas nas segunda e terceira sessões de tratamento. Para avaliar a efetividade dos dois tratamentos antes e após cada sessão, avaliaram-se: abertura bucal $(\mathrm{AB})$, por meio de régua milimetrada; dor, pela escala visual analógica; força de mordida (FM), usando um medidor de força digital; e qualidade de vida, avaliada imediatamente antes do início do tratamento e depois do término do tratamento. Resultados: a dor mostrou diferença estatística significativa no G2 após a segunda sessão $(p=0,020)$ e a terceira sessão $(p=0,047)$. Os demais resultados mostram que não houve diferença estatisticamente significativa entre os grupos $(p>0,05)$. Conclusão: considera-se que neste estudo piloto ambos os tratamentos foram eficazes para DTM muscular, uma vez que todos os pacientes apresentaram melhora dos sintomas. Não houve diferença estatisticamente significativa entre os tratamentos, exceto a dor pós-procedimento, causada pela técnica do agulhamento seco.

Palavras-chave: Agulhamento seco. Disfunção temporomandibular. Dor orofacial. Força de mordida.

\section{Introdução}

Um dos problemas mais comuns em nível de dor orofacial (DOF) é a disfunção temporomandibular (DTM), que corresponde a uma doença complexa, com caráter cíclico ou transitório, que envolve os músculos mastigatórios, a articulação temporomandibular (ATM) e suas estruturas ${ }^{1-4} \mathrm{e}$ é considerada também um grupo heterogêneo de problemas de saúde, por não possuir etiologia ou justificativa biológica. ${ }^{5}$ A DTM pode ser classificada como de origem articular, quando os sinais e sintomas estão associados à ATM, e de origem muscular, quando os sinais e sintomas estão associados aos músculos do sistema estomatognático. ${ }^{2,3}$

A DTM muscular apresenta um conjunto de sinais e sintomas, como: limitação na abertura bucal (AB), dor nos músculos, com presença de banda tensa e/ou ponto gatilho (PG), dor na cabeça, no pescoço e, até mesmo, nos dentes; consequentemente, a força de mordida (FM), dotada em Newton (N), também diminui. ${ }^{6}$ Além disso, essa patologia desencadeia prejuízos na qualidade de vida do indivíduo, ${ }^{7}$ pois causa impacto negativo nos pacientes, gerando prejuízo no trabalho, na escola, no sono e na alimentação. ${ }^{2,3,8}$ A severidade da doença gera um declive na qualidade de vida de forma diretamente proporcional. ${ }^{7,-11}$

http://dx.doi.org/10.5335/rfo.v23i2.8313

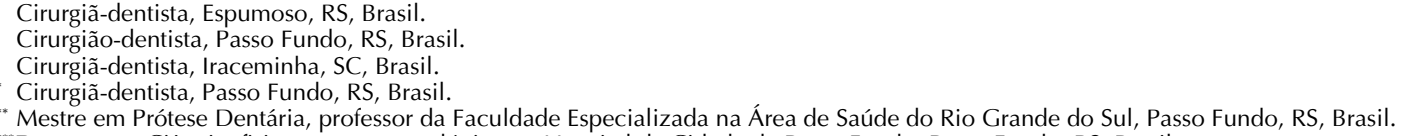


O tratamento correto da DTM muscular é consequência de um diagnóstico preciso. Cada caso deve ser tratado com a terapia mais simples e conservadora possível, tendo como objetivos reduzir as limitações, minimizar a dor e aumentar a qualidade de vida do paciente. O tratamento odontológico é efetivo para controlar a dor, quando associado a alongamento, exercícios ativos de relaxamento, massagem, farmacoterapia ou infiltração anestésica. ${ }^{8}$ Em concomitância, autores defendem que o tratamento com agulhamento seco é efetivo em pacientes portadores de DTM muscular, porque age diretamente no PG, eliminando o ácido lático e diminuindo a sintomatologia dolorosa. ${ }^{9,12,13}$

Os diferentes tratamentos para DTM caminham em desarmonia e nem sempre proporcionam a satisfação do paciente. Visando a elucidar o dentista e o paciente acerca do melhor caminho para o sucesso do tratamento da DTM muscular, o presente trabalho tem como objetivo comparar a eficiência de protocolos terapêuticos com e sem agulhamento seco.

\section{Sujeitos e método}

O presente trabalho trata de um estudo piloto de ensaio clínico randomizado. A amostra foi constituída por alunos do curso de Odontologia da Faculdade Especializada na Área de Saúde do Rio Grande do Sul (Fasurgs), maiores de 18 anos de idade, de ambos os gêneros e portadores de DTM muscular, que foram diagnosticados entre junho e setembro de 2015 e assinaram o termo de consentimento livre e esclarecido. Os alunos foram tratados a partir de agosto de 2015 na Clínica Odontológica da Fasurgs.

O presente trabalho foi autorizado pelo Comitê de Ética em Pesquisa (CEP) sob o parecer de n⿳ํㅡㄴ 1.143.351.

Fizeram parte da amostra dez indivíduos. Os estudantes portadores de DTM muscular foram selecionados por um pesquisador calibrado, por meio do questionário para avaliação da presença de sinais e sintomas para DTM recomendado pela Academia Europeia de Desordens Craniomandibulares, e diagnosticados pelo Critério Diagnóstico para Pesquisa em Disfunção Temporomandibular (Research Diagnostic Criteria for Temporomandibular Disorders - RDC/TMD). ${ }^{14}$ Após, os sujeitos foram randomizados e tratados pelos pesquisadores.

$\mathrm{O}$ pesquisador um realizou randomização dos alunos de forma aleatória, por meio de um sorteio com papéis coloridos colocados dentro de envelopes pardos, que se encontravam dentro de uma caixa. Os estudantes sabiam em qual grupo de tratamento estavam incluídos.

Os estudantes foram separados em dois grupos, conforme o protocolo de tratamento:

- Grupo 1 (G1): termoterapia com calor úmido (20 minutos), exercícios ativos de abertura e fechamento bucal com a ponta da língua encostada na face palatina dos incisivos superiores (três séries de 10 repetições) e massagem em deslizamento da origem para a inserção do músculo masseter (20 vezes) para relaxamento muscular, sendo essa sequência realizada três vezes ao dia;

- Grupo 2 (G2): o mesmo tratamento do G1 somado ao agulhamento seco.

$\mathrm{O}$ pesquisador dois orientou e supervisionou o protocolo de tratamento G1 para ambos os grupos quinzenalmente, na Fasurgs, durante três sessões. O protocolo também foi realizado diariamente, a nível domiciliar, por ambos os grupos.

O pesquisador três, docente da instituição e especialista em DTM/DOF, realizou o agulhamento seco no G2 na segunda e na terceira sessões de tratamento.

$\mathrm{O}$ pesquisador quatro avaliou os estudantes quinzenalmente na instituição, antes e depois de cada sessão de tratamento, ou seja, na pré-sessão (T1) e na pós-sessão (T2), por meio das mensurações da AB, da dor e da FM. Os dados pessoais e aqueles provenientes da avaliação foram transcritos para 0 formulário de coleta de dados.

$A$ mensuração da $A B$ foi realizada com régua específica, flexível, padronizada e escalada em milímetros, de uso individual (JON, Comércio de produtos odontológicos, São Paulo, São Paulo, Brasil). Os estudantes foram instruídos a realizar a $\mathrm{AB}$ máxima e mantê-la até o examinador terminar o procedimento.

A mensuração da dor foi realizada de acordo com a escala visual analógica, ${ }^{15}$ que se baseia em um quadro numerado de zero a dez e contém seis figuras de rosto ilustrativas, para ajudar a caracterizar a dor do estudante. O estudante escolheu o número e a figura que mais se assemelhava à sua dor.

A mensuração da FM foi realizada com um dinamômetro digital (Modelo DDK Kratos, Kratos Equipamentos Industriais Ltda., Cotia, Brasil), que fornece um valor em N. As superfícies do equipamento foram posicionadas sobre a face oclusal dos primeiros molares inferiores em ambos os lados e entre as faces incisais dos incisivos centrais superiores e inferiores. Para o teste, o estudante sentado ereto, com a cabeça estável, era instruído a morder o equipamento durante 10 segundos, com o máximo da força que fosse capaz de exercer. As medições foram realizadas em cada lado, por três vezes, com um intervalo de dois minutos. $\mathrm{O}$ valor final foi caracterizado pela média das medidas, com uma precisão de $0,01 \mathrm{~N}$.

Para caracterização da amostra, utilizaram-se estatísticas descritivas e percentagem, a fim de analisar as variáveis das perguntas em termos de frequência. Os dados com distribuição normal ( $\mathrm{AB}$ e FM) foram analisados por meio do teste $t$ de Student, para variáveis independentes para comparação entre os grupos, e do teste ANOVA, para análise em cada grupo ao longo do tempo. Naqueles com 
significância estatística, foi utilizada a análise de Post Hoc, por meio do teste de Tukey.

Os dados com distribuição não normal (dor) foram analisados por meio do teste de Mann-Withney, para comparação entre os grupos, e do teste de Kruskal-Wallis, para análise em cada grupo ao longo do tempo. Todas as análises foram realizadas utilizando o programa estatístico Bioestat 5.0, considerando $\mathrm{p} \leq 0,05$.

\section{Resultados}

Fizeram parte da amostra dez indivíduos, com

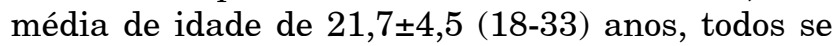
declaravam brancos, e nove $(90 \%)$ eram do gênero feminino. A pesquisa contou com a presença de dez estudantes em cada sessão de tratamento/avaliação, divididos em dois grupos de cinco alunos cada. $\mathrm{Na}$ primeira sessão, compareceu toda a amostra; porém, na segunda sessão, foram avaliados quatro em cada grupo, e, na terceira sessão, apenas três do G1 e cinco do G2.
No início de cada sessão, cada estudante foi questionado a respeito de como e quantas vezes estava realizando o tratamento caseiro. Foi constatado que nenhum dos alunos efetuou o tratamento da forma correta, pois oito deles realizavam duas vezes ao dia o procedimento, e dois realizavam apenas uma vez ao dia.

Analisando cada variável, em cada momento de avaliação, ao longo das sessões, observou-se que a $\mathrm{AB}$, no momento $\mathrm{T} 1$, apresentou uma oscilação entre os tempos, mas não houve diferença estatisticamente significativa; e, no momento T2, observou-se que o G1 apresentou uma diferença estatisticamente significativa na $A B$ entre as sessões $(p=0,006)$. Da mesma forma, ao observar a dor em cada grupo, em cada momento de avaliação, observou-se um aumento gradativo entre os tempos; entretanto, somente foi encontrada diferença significativa no G2 no T2 $(\mathrm{p}=0,010)$. Ao analisar a FM, observou-se que o G1 apresentou um aumento nos índices de FM ao longo das avaliações, e no G2 os valores variam entre os tempos, mas não houve diferença estatisticamente significativa (Tabela 1).

Tabela 1 - Avaliação das variáveis entre as sessões no momento T1 e T2

\begin{tabular}{|c|c|c|c|c|c|c|c|c|}
\hline & Grupo & $\mathrm{N}$ & 1ํㅗ Sessão & $\mathrm{N}$ & $2^{\text {a }}$ Sessão & $N$ & $3^{a}$ Sessão & $\mathrm{p}$ \\
\hline \multirow[t]{4}{*}{ Abertura bucal } & T1 G1 & 5 & $42,8( \pm 8,5)$ & 4 & $43,0( \pm 5,3)$ & 3 & $41,7( \pm 9,0)$ & 0,972 \\
\hline & T1 G2 & 5 & $45,2( \pm 4,0)$ & 4 & $48,7( \pm 4,7)$ & 5 & $45,0( \pm 8,0)$ & 0,598 \\
\hline & T2 G1 & 5 & $44,0( \pm 4,8)$ & 4 & $51,5( \pm 2,3)$ & 3 & $40,0( \pm 2,0)$ & 0,006 \\
\hline & T2 G2 & 5 & $48,8( \pm 4,6)$ & 4 & $46,2( \pm 3,8)$ & 5 & $38,8( \pm 9,9)$ & 0,104 \\
\hline \multirow[t]{4}{*}{ Dor } & T1 G1 & 5 & $1,6( \pm 1,8)$ & 4 & $2,0( \pm 2,4)$ & 3 & $2,7( \pm 1,2)$ & 0,739 \\
\hline & T1 G2 & 5 & $1,4( \pm 1,9)$ & 4 & $1,5( \pm 3,0)$ & 5 & $2,0( \pm 2,8)$ & 0,924 \\
\hline & T2 G1 & 5 & $1,6( \pm 2,2)$ & 4 & $1,3( \pm 1,9)$ & 3 & $2,0( \pm 1,7)$ & 0,974 \\
\hline & T2 G2 & 5 & $0,8( \pm 0,8)$ & 4 & $7,5( \pm 1,7)$ & 5 & $6,4( \pm 3,0)$ & 0,010 \\
\hline \multirow[t]{4}{*}{ Força de mordida } & T1 G1 & 5 & $251,2( \pm 141,7)$ & 4 & $251,8( \pm 143,3)$ & 3 & $267,3( \pm 55,4)$ & 0,983 \\
\hline & T1 G2 & 5 & $292,8( \pm 155,8)$ & 4 & $261,8( \pm 120,2)$ & 5 & $271,5( \pm 128,2)$ & 0,940 \\
\hline & T2 G1 & 5 & $241,2( \pm 126,7)$ & 4 & $243,8( \pm 105,1)$ & 3 & $278,3( \pm 67,8)$ & 0,695 \\
\hline & T2 G2 & 5 & $313,8( \pm 135,5)$ & 4 & $150,3( \pm 61,8)$ & 5 & $175,4( \pm 100,6)$ & 0,150 \\
\hline
\end{tabular}

Fonte: autores.

Comparando as variáveis entre os dois grupos, em cada tempo de avaliação, não se verificou diferença estatisticamente significativa na $\mathrm{AB}$ na pré-sessão e na pós-sessão. Contudo, a dor foi signifi- cativamente diferente no G2 após a segunda sessão $(\mathrm{p}=0,020)$ e a terceira sessão $(\mathrm{p}=0,047)$. Na FM, também não se observou diferença estatisticamente significativa entre os grupos (Tabela 2). 
Tabela 2 - Comparação das variáveis entre os grupos de tratamento em cada tempo de avaliação

\begin{tabular}{|c|c|c|c|c|c|c|}
\hline & Sessão & $\mathrm{N}$ & G1 & $\mathrm{N}$ & G2 & $p$ \\
\hline \multirow[t]{6}{*}{ Abertura bucal } & $1^{\mathrm{a}} \mathrm{T} 1$ & 5 & $42,8( \pm 8,5)$ & 5 & $45,2( \pm 4,0)$ & 0,593 \\
\hline & $1 \stackrel{\mathrm{a}}{\mathrm{T} 2}$ & 5 & $44,0( \pm 4,8)$ & 5 & $48,8( \pm 4,6)$ & 0,147 \\
\hline & $2^{\mathrm{a}} \mathrm{T} 1$ & 4 & $43,0( \pm 5,3)$ & 4 & $48,7( \pm 4,7)$ & 0,159 \\
\hline & $2^{\mathrm{a}} \mathrm{T} 2$ & 4 & $51,5( \pm 2,3)$ & 4 & $46,2( \pm 3,8)$ & 0,069 \\
\hline & $3^{\mathrm{a}} \mathrm{T} 1$ & 3 & $41,7( \pm 9,0)$ & 5 & $45,0( \pm 8,0)$ & 0,628 \\
\hline & $3^{\mathrm{a}} \mathrm{T} 2$ & 3 & $40,0( \pm 2,0)$ & 5 & $38,8( \pm 9,9)$ & 0,805 \\
\hline \multirow[t]{6}{*}{ Dor } & $1^{\mathrm{a}} \mathrm{T} 1$ & 5 & $1,6( \pm 1,8)$ & 5 & $1,4( \pm 1,9)$ & 0,736 \\
\hline & $1^{\mathrm{a}} \mathrm{T} 2$ & 5 & $1,6( \pm 2,2)$ & 5 & $0,8( \pm 0,8)$ & 0,911 \\
\hline & $2^{\mathrm{a}} \mathrm{T} 1$ & 4 & $2,0( \pm 2,4)$ & 4 & $1,5( \pm 3,0)$ & 0,741 \\
\hline & $2^{\mathrm{a}} \mathrm{T} 2$ & 4 & $1,3( \pm 1,9)$ & 4 & $7,5( \pm 1,7)$ & 0,020 \\
\hline & $3^{\mathrm{a}} \mathrm{T} 1$ & 3 & $2,7( \pm 1,2)$ & 5 & $2,0( \pm 2,8)$ & 0,536 \\
\hline & $3^{\mathrm{a}} \mathrm{T} 2$ & 3 & $2,0( \pm 1,7)$ & 5 & $6,4( \pm 3,0)$ & 0,047 \\
\hline \multirow[t]{6}{*}{ Força de mordida } & $1^{\mathrm{a}} \mathrm{T} 1$ & 5 & $251,2( \pm 141,7)$ & 5 & $292,8( \pm 155,8)$ & 0.672 \\
\hline & $1 \stackrel{\mathrm{a}}{\mathrm{T} 2}$ & 5 & $241,2( \pm 126,7)$ & 5 & $313,8( \pm 135,5)$ & 0,407 \\
\hline & $2^{\mathrm{a}} \mathrm{T} 1$ & 4 & $251,8( \pm 143,3)$ & 4 & $261,8( \pm 120,2)$ & 0,916 \\
\hline & $2^{\mathrm{a}} \mathrm{T} 2$ & 4 & $243,8( \pm 105,1)$ & 4 & $150,3( \pm 61,8)$ & 0,187 \\
\hline & $3^{\mathrm{a}} \mathrm{T} 1$ & 3 & $267,3( \pm 55,4)$ & 5 & $271,5( \pm 128,2)$ & 0,953 \\
\hline & $3^{\mathrm{a}} \mathrm{T} 2$ & 3 & $278,3( \pm 67,8)$ & 5 & $175,4( \pm 100,6)$ & 0,137 \\
\hline
\end{tabular}

Fonte: autores.

\section{Discussão}

A DTM não apresenta etiologia definida, sendo multifatorial, relacionada a diversos fatores fisiopatológicos..$^{2-4,6}$ A DTM muscular tem incidência entre 15 e 30 anos de idade, ${ }^{1,16}$ sendo sua prevalência, geralmente, de quatro mulheres para um homem, ${ }^{1,3,5}$ uma vez que os estudos mostram que as mulheres em idade fértil são mais vulneráveis geneticamente a etiologia, em função das taxas hormonais. ${ }^{4}$

O paciente com DTM pode apresentar desconforto, função mandibular limitada, abertura bucal prejudicada, dificuldade de morder e cortar os alimentos, dores que irradiam para a mandíbula, a ATM e/ou a cabeça e dor à palpação muscular e articular, ${ }^{1-5,8,17}$ sendo que a dor à palpação está intimamente ligada à presença de PG no interior do músculo. ${ }^{18}$

$\mathrm{O}$ presente estudo averiguou os sinais e sintomas e diagnosticou a presença de DTM muscular nos estudantes avaliados por meio do RDC/TMD, corroborando com estudos semelhantes encontrados na literatura. ${ }^{7,9-12,19,20}$
Este estudo observou que todos os pacientes apresentavam dores musculares e mais de um PG no músculo masseter bilateralmente, corroborando com estudos encontrados na literatura. ${ }^{9,12,21}$

O paciente portador de DTM procura tratamento, a fim de eliminar sua sintomatologia. O cirurgião-dentista deve propor terapias que aliviem e/ou eliminem a dor, restaurem a função e previnam a recorrência. ${ }^{2,17}$ Deve ainda orientar o paciente a modificar os hábitos orais, para complementar o tratamento da DTM muscular, às vezes necessitando de um terapeuta para auxiliar a mudança de hábito necessária. ${ }^{1}$

O tratamento para DTM deve condizer com um procedimento simples, reversível, não invasivo e de baixo custo. ${ }^{17}$ Mas a literatura apresenta uma vasta gama de terapias indicadas ao tratamento da DTM muscular ou DOF; entre as terapias encontradas, as mais indicadas, de acordo com a efetividade, são: termoterapia com calor úmido, exercícios mandibulares e massagem.

A hipertermia é uma terapia não invasiva e de baixo custo, não proporciona risco de iatrogenia, di- 
minui a dor e a inflamação local e aumenta a flexibilidade muscular. ${ }^{22}$

Pacientes portadores de DTM muscular que realizaram o tratamento com exercícios terapêuticos de $\mathrm{AB}$ obtiveram eficácia a longo prazo, pois reduziu a dor, e melhoram a função muscular e a qualidade de vida. A maioria dos estudos associou o exercício a outras terapias para DTM, e uma minoria tratou tal patologia com exercícios terapêuticos de $\mathrm{AB}$ sem associação com outras terapias. ${ }^{16}$

A massagem realizada no masseter libera endorfina, aliviando a dor física, e, quando associada a outras terapias não invasivas, potencializa o efeito. ${ }^{23}$

Por esses motivos, no presente estudo, optou-se por associar as terapias para os grupos em tratamento, que receberam hipertermia induzida, exercícios bucais e massagem. Esse conjunto de terapias produziu efeito muito positivo em relação ao controle de DTM muscular, apresentando aumento da FM e da $\mathrm{AB}$ máxima sem dor, bem como diminuição significativa da dor; houve ainda uma pequena porcentagem que erradicou completamente a dor.

Cabe ressaltar que cada estudante foi motivado à realização dos exercícios em domicílio, devido ao fato de a patologia crônica necessitar de tratamento constante, corroborando com as indicações de estudos encontrados na literatura. ${ }^{16}$

Porém, os estudantes deste estudo relataram que não realizaram o tratamento domiciliar como foi orientado e proposto a eles. Contudo, se os pacientes tivessem colaborado mais, realizando o protocolo proposto, a terapia teria dado resultado ainda maior.

Em contraponto, a terapia de agulhamento seco, que é minimamente invasiva, vem ganhando campo e se desenvolvendo como um importante trabalho frente ao tratamento da DTM muscular, pois age diretamente no PG, eliminando o ácido lático e minimizando os sinais e sintomas da doença, ${ }^{9,12,13}$ além de aumentar $\mathrm{a} \mathrm{AB}^{9}$ e reduzir a intensidade e a interferência da dor sob a qualidade de vida dos pacientes. ${ }^{12}$

As modalidades terapêuticas menos invasivas foram utilizadas em ambos os grupos do presente estudo, e a terapia minimamente invasiva foi utilizada apenas no G2, a fim de verificar a efetividade de tais protocolos, pois nenhum artigo da literatura levantada comparou um tratamento não invasivo com um minimamente invasivo. Os relatos na literatura são na maioria estudos que avaliam um tratamento isoladamente ou comparam-no com um grupo controle, que não recebe nenhum tipo de tratamento. ${ }^{12,16,21}$ Outros autores avaliaram várias terapias de forma associada, porém todas não invasivas $^{23}$ ou todas minimamente invasivas. ${ }^{9,13}$

Para constatar a real efetividade de qualquer tratamento é necessária a realização de avaliações criteriosas dos sinais e sintomas do paciente. Este estudo, então, propôs a avaliação da $\mathrm{AB}$, da dor e da
FM antes e depois do tratamento para DTM muscular, corroborando com a literatura. ${ }^{21}$

Para a avaliação da $\mathrm{AB}$ máxima sem dor, o presente estudo optou pela utilização do método mais acessível economicamente para a técnica, a régua milimetrada flexível, em concordância com os estudos encontrados na literatura. ${ }^{9}$

Em T1, a AB em ambos os grupos teve uma oscilação de medidas, com maior abertura na segunda sessão, mas essa diferença não foi estatisticamente significativa. Já no T2, houve uma diferença estatisticamente significativa ( $p=0,006)$ no G1; foi, então, realizada a análise de Post Hoc, para evidenciar a maior diferença estatisticamente significativa entre a segunda e a terceira sessões $(p=0,006)$, pois a diferença entre a primeira e a segunda sessão também foi estatisticamente significativa $(\mathrm{p}=0,032)$.

A diferença significativa encontrada pode ser decorrente da grande mudança de $\mathrm{AB}$ logo após a segunda sessão de tratamento, tendo em vista a grande expectativa do paciente frente ao tratamento. Além disso, estima-se que a diferença pode se dar em função de os estudantes terem saído da condição de nenhum tratamento para uma terapia caseira de três sessões diárias. Há ainda o fato de, na última sessão, o número de estudantes ter diminuído consideravelmente, não sendo possível chegar a uma comparação tão fidedigna quanto necessário.

Um estudo que associou $\mathrm{AB}$ e tratamento de agulhamento seco, ${ }^{9}$ constatou aumento da $\mathrm{AB}$ com a técnica minimamente invasiva. Cabe ressaltar que apenas duas sessões de agulhamento seco são limitantes à construção de um resultado fidedigno, mas se trata de um estudo piloto, em que a coleta continuará a longo prazo.

No presente estudo, a $\mathrm{AB}$, quando comparada entre os dois grupos, em cada tempo de avaliação, encontrou-se maior no G2 na maioria dos tempos, com exceção das duas sessões no T2, em que o grupo recebeu agulhamento; porém essa diferença não apresentou valores estatisticamente significativos para ambos os grupos. Nesse caso, explica-se a rigidez muscular decorrida do quadro de fadiga muscular. $^{24}$

Para a mensuração da dor, o presente estudo utilizou como instrumento de avaliação a escala visual analógica, vastamente utilizada na literatura, pois traz à pesquisa uma informação precisa sobre a dor do paciente, indo ao encontro de outros estudos. ${ }^{9,12,13,16,20,21}$

Em ambos os grupos, em T1, os estudantes chegaram a cada sessão de tratamento com um pouco mais de dor do que na sessão anterior, porém esse efeito não foi estatisticamente significativo. Já em T2, em ambos os grupos, observou-se uma oscilação ao longo das sessões, após a aplicação das terapias; entretanto, apenas a diferença no G2 foi estatisticamente significativa $(p=0,10)$. A partir da segunda sessão, todos os estudantes deste grupo receberam 
agulhamento seco no músculo masseter, aumentando a dor momentânea, o que explica o fato.

Os artigos consultados não apresentam o histórico de dor entre as sessões de tratamento não invasivo, apontam apenas diminuição da dor ao final do tratamento. ${ }^{16}$ Os estudos de tratamentos minimamente invasivos afirmam que a dor do paciente diminui ao final do tratamento. ${ }^{9,12,13}$

A dor, neste estudo, quando comparada entre os dois grupos, em cada tempo de avaliação, não mostrou diferenças significativas na maioria dos tempos. Contudo, constatou-se diferença estatisticamente significativa no G2 após a segunda sessão $(p=0,020)$ e após a terceira sessão $(p=0,047)$. Essa diferença justifica-se pelo fato de que, a partir da segunda sessão, no G2, houve abordagem com agulhamento, corroborando com estudos encontrados na literatura. ${ }^{25}$

Para a mensuração da FM, o presente estudo utilizou um dinamômetro digital específico para a avaliação, seguindo alguns estudos anteriores, pois o custo-benefício é mais relevante. ${ }^{20,21}$

Alguns autores avaliaram a FM apenas na presença de DTM em várias faixas etárias; ${ }^{26-30}$ e outros estudos que avaliaram a força apenas em pacientes saudáveis ${ }^{28,31,32}$ obtiveram FM de $150 \mathrm{~N}$ de força unilateral em primeiro molar ${ }^{32}$ e FM máxima em homens saudáveis de $300 \mathrm{~N}$ e em mulheres saudáveis de $210 \mathrm{~N} .{ }^{28}$

No presente estudo, a FM, quando comparada entre os dois grupos, em cada tempo de avaliação, foi maior no G2, na maioria dos tempos, com exceção da segunda e da terceira sessão, ambas em T2; essa diferença não apresentou valores estatisticamente significativos para ambos os grupos. Observou-se que o paciente com maior dor apresentou menor FM, corroborando com a literatura. ${ }^{27,29,30}$

O presente trabalho demonstrou que a FM média dos estudantes com DTM, sem terem realizado anteriormente algum tipo de tratamento para essa patologia, variou de $251,2 \mathrm{~N}$ a $292,8 \mathrm{~N}$, corroborando com a literatura. ${ }^{26,28}$

A fim de avaliar a interferência da dor sobre a FM, um estudo induziu pacientes saudáveis a dor e concluiu que, de acordo com os resultados obtidos, o pico de dor aumentou após a injeção, porém a dor provocada não alterou a FM máxima voluntária. ${ }^{20}$

$\mathrm{O}$ presente estudo encontrou como dados de FM, no T1, um aumento na força do G1 e uma oscilação de medidas no G2, mas essas diferenças não foram estatisticamente significativas. Já no T2, o G1 aumentou a força ao longo do tempo, logo após a aplicação do tratamento, e o G2 diminuiu a força na segunda sessão (início do tratamento com agulhamento seco) e passou a aumentar na terceira sessão; contudo esses valores não caracterizaram diferença estatisticamente significativa. Analisando os dados coletados, supõe-se que a FM em ambos os grupos aumenta após mais sessões de tratamento, bem como realiza uma avaliação após decorridos alguns dias do ápice da sintomatologia dolorosa desencadeada pelo agulhamento seco, uma vez que a literatu- ra constata que a FM do paciente aumenta no final do tratamento para DTM, quando este é realizado de forma bem efetiva. ${ }^{21,33}$

Estima-se que não houve diferença estatisticamente significativa na maioria das variáveis analisadas, em função de o tempo de tratamento ser razoavelmente curto. Além disso, a amostra foi restrita, devido ao grande índice de não comparecimento às sessões, principalmente na última sessão, visto que os sinais e sintomas álgicos já não eram mais tão evidentes quanto no começo do tratamento.

Este estudo apresenta limitações, mas, por se tratar de um estudo piloto, os resultados ainda serão melhor analisados com a finalização do trabalho, permitindo uma verificação em longo prazo e com um número de pacientes maior.

\section{Conclusão}

Por meio do estudo realizado, considera-se que ambos os tratamentos foram eficazes para DTM muscular, uma vez que todos os pacientes apresentaram melhora dos sintomas. Não houve diferença estatisticamente significativa entre os tratamentos, exceto a dor pós-procedimento, causada pela técnica do agulhamento seco.

\section{Abstract}

Objective: the present study aims to compare the efficacy of two treatment protocols for temporomandibular dysfunction (TMD) of muscular origin. Subjects and method: students of the Faculdade Especializada na área de Saúde do Rio Grande do Sul, that were selected through the questionnaire to evaluate the presence of TMD signs and symptoms and diagnosis of muscular TMD from the Diagnostic Criteria for Temporomandibular Research Disorders. Ten students composed the sample, divided into two groups according to the treatment protocol: hyperthermia induced by G1, mandibular exercises and massage; G2 $\square$ dry needling, induced hyperthermia, mandibular and massage exercises. Students were instructed to complete the G1 treatment protocol every day at home and at the educational institution for three sessions that occurred every fifty days. Dry needling was performed in G2 only in the second and third treatment sessions. To evaluate the effectiveness of the two treatments before and after each session, we evaluated: mouth opening $(A B)$, through a millimeter ruler; visual analogue scale; bite force (FM) using a digital force gauge and quality of life was assessed immediately prior to initiation of treatment and after termination of treatment. Results: pain obtained a statistically significant difference in $\mathrm{G} 2$ after the second session $(p=0.020)$ and the third session $(p=0.047)$. The other results show that there was no statistically significant difference between the groups ( $p>0.05)$. Conclusion: it is considered that in this pilot study both treatments were effective for muscular TMD, since all the patients presented improvement of the symptoms. There was no statistically significant difference between treatments, except for post-procedure pain, caused by the dry needling technique.

Keywords: Dry needling. Disorders temporomandibular. Orofacial pain. Bite force. 


\section{Referências}

1. Bove SRK, Guimarães AS, Smith RL. Caracterização dos pacientes de um ambulatório de disfunção temporomandibular e dor orofacial. Rev Latino-Am Enfermagem 2005; 13(5):686-91.

2. Carrara SV, Conti PCR, Barbosa JS. Termo do $1^{\circ}$ Consenso em Disfunção Temporomandibular e Dor Orofacial. Dental Press J Orthod 2010; 15(3):114-20.

3. Donnarumma MDC, Muzilli CA, Ferreira C, Nemr K. Disfunções Temporomandibulares: sinais, sintomas e abordagem multidisciplinar. Rev CEFAC 2010; 12(5):788-94.

4. Sartoretto SC, Bello YD, Bona AD. Evidências científicas para o diagnóstico e tratamento da DTM e a relação com a oclusão e a ortodontia. Rev Fac Odontol Univ Passo Fundo 2012; 17(3):352-9.

5. Branco RS, Branco CS, Tesch, RS, Rapoport A. Frequência de relatos de parafunções nos sub-grupos diagnósticos de DTM de acordo com os critérios para pesquisa em disfunções temporomandibulares (RDC/TMD). Rev Dental Press Ortodon Ortop Facial 2008; 13(2):61-9.

6. Santos ECA, Bertoz FA, Pignatta LMB, Arantes FM. Avaliação clínica de sinais e sintomas da disfunção temporomandibular em crianças. R Dental Press Ortodon Ortop Facial 2006; 11(2):29-34.

7. Barros VM, Seraidarian PI, Côrtes MIS, De Paula LV. The impacto $f$ oraficial pain on the quality of life of patients temporomandibular disorders. J Orofac Pain 2009; 23:28-37.

8. Figueiredo VMG, Cavalcanti AL, Farias ABL, Nascimento SR. Prevalência de sinais, sintomas e fatores associados em portadores de disfunção temporomandibular. Acta Scientiarum Health Sciences 2009; 31(2):159-63.

9. Fernandez-Carnero J, La Touche R, Ortega-Santiago R, Galan-del-Rio F, Pesquera J, Ge HY, et al. Short-Term Effects of Dry Needling of Active Myofascial Trigger Points in the Masseter Muscle in Patients With Temporomandibular Disorders. J Orofac Pain 2010; 24:106-12.

10. Meulen MJ, John MT, Naeije M, Lobbezoo F. Devoloping abbreviated OHIP versions for use with TMD patients. J Oral Rehabil 2012; 39(1):18-27.

11. Miettinen O, Lahti S, Sipilã K. Psychosocial aspects of temporomandibular disorders and oral health-related quality-oflife. Acta Odontol Scandinavica 2012; 70(1):331-6.

12. Huang YT, Lin SY, Neoh CA, Wang KY, Jean YH, Shi HY. Dry needling for myofascial pain: prognostic factors. J Altern Complement Med 2011; 18(8):755-62.

13. McMillan AS, Nolan A, Kelly PJ. The efficacy of dry needling and procaine in the treatment of myofascial pain in the jaw muscles. J Orofac Pain 1997; 1(14):307-14.

14. Dworkin SF, LeResche L. Research diagnostic criteria for temporomandibular disorders: review, criteria, examinations and specifications, critique. J Craniomandib Disord 1992; 6:301-55.

15. Price DD, McGrath PA, Rafii A, Buckingham B. The validation of visual analogue scales as ratio scale measures for chronic and experimental pain. Elsevier Science Publishers B V 1983; 1:45-56.

16. Maluf AS, Moreno BGD, Alfredo PP, Marques AP, Rodrigues G. Exercícios terapêuticos nas desordens temporomandibulares: uma revisão de literatura. Fisioter Pesq 2008; 15(4):408-15.

17. Franco AL, Zamperini CA, Salata DC, Silva EC, Albino Júnior W, Camparis CM. Fisioterapia no tratamento da dor orofacial de pacientes com disfunção temporomandibular crônica. Rev Cubana Estomatol 2011; 48(1):56-61.

18. Caramêz J, Carvalhão F, Real Dias MC. Dor miofascial por pontos gatilho, uma doença multidisciplinar. Acta Reumatol Port 2009; 34:38-43.
19. Almoznino G, Zini A, Zakuto A, Sharav Y, Haviv Y, Avraham $\mathrm{H}$, et al. Oral health-related quality of life in patients with temporomandibular disorders. J Oral Facial Pain Headache 2015; 29:231-41.

20. Kumar A, Castrillon EC, Svensson P. Can Experimentally Evoked Pain in the Jaw Muscles or Temporomandibular Joint Affect Anterior Bite Force in Humans? J Oral Facial Pain Headache 2015; 29(1):31-40.

21. Dias LM, Spinato IL, Vasconcelos TB, Rodrigues DV, Macena CBA, Farias MSQ, et al. Effects of muscle inhibition technique on relief of masticatory pain in patients with temporomandibular disorders: an experimental study. Con Scientiae Saúde 2013; 12(2):298-304.

22. Felice TD, Santana LR. Recursos Fisioterapêuticos (Crioterapia e Termoterapia) na espasticidade: revisão de literatura. Rev Neurocienc 2009; 17(1):57-62.

23. Santos LFS, Pereira MCA. A efetividade manual no tratamento de disfunções temporomandibulares (DTM): uma revisão de literatura. Rev Aten Saúde 2016; 14(49):72-7.

24. Watanabel M, Tabata T, Huh JI, Inai T, Tsuboi A, Sasaki $\mathrm{K}$, et al. Possible Involvement of Histamine in Muscular Fatigue in Temporomandibular Disorders: Animal and Human Studies. J Dent Res 1999; 78(3):769-75.

25. Venancio RA, Alencar FGP, Zamperini C. Botulinum Toxin, Lidocaine, and Dry-Needling Injections in Patients with Myofascial Pain and Headaches. The J Craniomandibular Practice 2009; 27(1):46-53.

26. Hansdottir R, Bakke M. Joint tenderness, jaw opening, chewing velocity, and bite force in patients with temporomandibular joint pain and matched healthy control subjects. J Orofac Pain 2004; 18(2):108-13.

27. Kogawa EM, Calderon PS, Lauris JRP, Araujo CRP, Conti PCR. Evaluation of maximal bite force in temporomandibular disorders patients. J Oral Rehabil 2006; 33(8):559-65.

28. May B, Saha S, Saltzman M. A three-dimensional mathematical model of temporomandibular joint loading. Clin Biomech 2001; 16(6):489-95.

29. Sonnesen L, Bakke M. Molar bite force in relation to occlusion, craniofacial dimensions, and head posture in pre-orthodontic children. European J Orthodontics 2005; 27(1):58-63.

30. Sonnesen L, Bakke M, Solow B. Bite force in pre-orthodontic children with unilateral crossbite. Eur J Orthodontics 2001; 23(6):741-9.

31. Mountain G, Wood D, Toumba J. Bite force measurement in children with primary dentition. Int J Paediatr Dent 2011; $2(21): 112-8$.

32. Shimada A, Yamabe Y, Torisu T, Baad-Hansen L, Murata H. Measurement of dynamics bite force during mastication. J Oral Rehabil 2012; 5(39):349-56

33. Aguiar ASW, Oliveira ACX, Martins PC, Freire ROM. Avaliação do grau de abertura bucal e dor pós-operatória após a remoção de terceiros molares inferiores retidos. Rev Cir Traumatol Buco-Maxilo-Fac 2005; 5(3):57-64.

\section{Endereço para correspondência:}

\section{Júlia Pagnussat}

Rua Salvador Coser, 77, São Jorge 994000-000, Espumoso, RS, Brasil Telefone: (54) 99933-9058

E-mails: ju_pagnussat@hotmail.com leo77saraiva@hotmail.com

Recebido: 25/09/18. Aceito: 22/10/18. 